\title{
Motion Simulation in a Virtual Basketball Shooting Teaching System
}

\author{
http://dx.doi.org/10.3991/ijoe.v12i02.5049 \\ CHEN Gong ${ }^{1,2}$, CHEN Ning ${ }^{3}$ \\ ${ }^{1}$ Wuhan University of tenology, Wuhan, China \\ ${ }^{2}$ Guangxi Economic \& Trade Polytechnic, Guangxi, China \\ ${ }^{3}$ Wuhan Textile University, Wuhan, China
}

\begin{abstract}
With the development and popularity of basketball, basketball technology requires good methods to spread related technology, so more and more people can master basketball and take part in the joy of the basketball experience. With the new technologies and methods emerging in regard to shooting a basketball, the characteristics for understanding and mastering the principles of basketball should be researched. This paper, uses motion simulation in a virtual basketball shooting teaching system to simulate the process of moving basketball players to provide scientific reference data.
\end{abstract}

Index Terms-Simulation; Virtual System; Basketball teaching

\section{INTRODUCTION}

Virtual reality can provide many kinds of feedback for users, including visual, auditory, haptic, etc. These feedback technologies can improve users' evaluation of the sense of presenting, which is provided by simulation systems of virtual reality. These experiences can enhance the performance of subjects who completed the test task in a simulation environment. At the same time, with the development of digital human modeling technology, a virtual reality simulation system has emerged that can help users be immersed and personally experience interacting with the objects in the virtual environment and put it into actual research and evaluation. A virtual reality sports simulation system is composed of virtual reality technology and interactive sports simulation, which has reference value in theory and a certain significant meaning for improving the training level of the athletes.

A virtual reality P.E. teaching system generally includes a video image that is projected on a screen and audio information that is played synchronically [1-4]. In addition, using sensors can make virtual reality sports simulation systems monitor the practical performance of the objects in real time and capture relevant data. Then a computer is used to analyze the data acquired by the sensors, providing corresponding feedback to the object's behavior accordingly [5]. The current virtual reality P.E. teaching system integrates these functions into one, providing users with a real sports competition environment in a virtual reality environment, adding elements such as visual and auditory simulation, etc, and allowing the athletes or sports participants, etc. to obtain real sports experience [6].

In basketball, shooting is the only means of scoring, which is the focus of the game as well as the core of con- trolling the ball for the two sides. In order win, players should improve the hit rate of shooting; therefore, basketball teaching and training includes a lot of shooting and practice time. To improve effectiveness, the teaching and training needs scientific and reasonable methods. The scientific and reasonable methods and means should be built on the basis of understanding the essence of shooting techniques by observing and providing feedback on the shooting practice. It can determine how many mistakes beginners make in shooting. From the surface, it is a phenomenon of generalization technology, but the root of the mistake is that the beginners do not understand how to shoot correctly and are not clear what criteria to test whether the shooting technique is correct. Students only know what it is, but they do not know why it is. In training and teaching, they are only asked to put emphasis on the action or simply imitate the demonstration action. By using such mechanical training and form, it is easy for the students to make all kinds of wrong actions without knowing it. By the time the problem is found, it has wasted a lot of time and needs more time and energy to correct the mistakes, which results in low efficiency in training and learning. Therefore, exploring a method for improving the efficiency of the shooting training and for teaching basketball shooting, virtual simulation technology is the purpose of this thesis.

\section{BALl ANimation Algorithm Of VirtuAl SHOOTING}

This section studies the ball's motion animation in the large scale scene. A spherical object is a kind of basic geometric object with broad representation. The virtual shooting system required studying the elastic deformation of the spherical surface and parabolic motion animation of spherical object. Elastic deformation is a common pattern of deformation. After the object experiences deformation, it can restore the original shape according to the elastic model. The algorithm of elastic deformation is based on a particle system; at the same time, a gridless method is adopted to improve the efficiency of the calculation of deformation. It makes each vertex of the dimensional objects that formed the three-dimensional deformation a particle; thus, the deformed object can represent a particle system. By controlling the particle, it can also change the shape of the object. At the same time, it sets up a corresponding target position for each particle that consists of the deformation objects. After the deformation occurs, the elastic power will pull the particles to the target position so the deformed object can restore the original shape. This thesis dealt with the deformed algorithm, a three- 
dimensional object composed by the particle with quality and initial position. Because there is no connected information between each particle, it did not consider the interaction between the particles; it only needed to consider the collision of the particle and environment as well as the external force of the particle. It was assumed that the corresponding relationship was known between the initial shape and the actual shape of the object. Therefore, the main task was to determine the optimal rigid transformation of the two point clouds. The problem can be stated as follows: for the two groups of given points: $x i$ and $x 0$, calculate the rotation matrix $\mathrm{R}$ and the translation vector $\mathrm{t}$ and $\mathrm{t} 0$, minimizing the formula below:

$$
\sum_{i} w_{i}\left(R\left(x_{i}^{0}-t_{0}\right)+t-x_{i}\right)^{2}
$$

Among them, mi is the weight value of each point, and apparently the optimal translation vector is the centroid of the initial shape and the actual shape, i.e.:

$$
t_{0}=x_{c m}^{0}=\frac{\sum_{i} m_{i} x_{i}^{0}}{\sum_{i} m_{i}}, t=x_{c m}=\frac{\sum_{i} m_{i} x_{i}}{\sum_{i} m_{i}}
$$

Among them, Tvv is a symmetric matrix, containing only scaling without rotation. Therefore, the optimum rotary torque array $\mathrm{R}$ is the rotating part of matrix Tuv, through the polar decomposition $T_{u v}=R \cdot S$, R can be obtained, among them, $S=\sqrt{T_{u v}^{T} T_{u v}}$ is the symmetric part, $R=T_{U V} S^{-1}$ is the rotating part, the final target position can be calculated by the following formula:

$$
g_{i}=R\left(x_{i}^{0}-x_{c m}^{0}\right)+x_{c m}
$$

Among them, a is the parameter of the object's hardness, fext is the external force on the particle. According to the algorithm of elastic deformation, it can realize the simulation of object's deformation.

$$
\begin{aligned}
& v_{i}=(t+\Delta t)=v_{i}(t)+a \frac{g_{i}(t)-x_{i(t)}}{\Delta t}+\Delta t \cdot f_{e x t}(t) / m_{i} \\
& x_{i}(t+\Delta t)=x_{i}(t)+\Delta t \cdot v_{i}(t+\Delta t)
\end{aligned}
$$

\section{Check The Algorithm Of Elastic DEFORMATION}

The deformed object used here is the $3 \mathrm{D}$ model of a basketball; from the left to the right is the process of the ball falling from the sky to the ground, colliding and deforming, bouncing back into the air, and restoring to its original shape. By using the algorithm of elastic deformation proposed in this thesis to simulate the deformation of basketball, after the deformation of the sphere, it can be recovered to its original shape. The following describes the performance of the algorithm of elastic deformation. It can be seen, as the number of particles of the deformed object is increased, the rendering speed is decreased. This is because the increased size of the particles requires more resources to calculate the deformation, but compared with the calculation of not adopting the deformation algorithm, adopting the algorithm of deformation does not make the rendering speed drop a lot. This is because in this thesis it adopts a gridless method, which does not need to consider the interaction between the particles. Thus, it reduces the complexity and improves the efficiency of the calculation.
Therefore, the algorithm of elastic deformation can be enough to meet the need of real-time rendering.

According to analysis of the virtual shooting of the basketball, basketball games are usually held indoors, so there is no interference of wind and rain. The influence of the power from the gravity and air resistance in the process of basketball's rapid flight is far greater than the effect of the buoyancy of the air and air pressure. Therefore, it can ignore the influence factors of air buoyancy and air pressure. Secondly, the moving distance of the basketball is much smaller than the radius of the earth. So it can be assumed that the surface of the earth is flat rather than spherical, and it can ignore the Coriolis inertial force caused by the rotation of the earth. Finally, assuming that the shape of the basketball is spherical with standard axial symmetry during the flight, the effect of the air friction on the speed of basketball can also be ignored.

The motion model proposed in this thesis is mainly considering the effect of gravity and the air resistance on the basketball. Through analyzing the force of gravity and the air resistance, the flight trajectory of the basketball is a parabola. The parabola trajectory of the basketball and the final landing point are dependent on the initial velocity vector, the fingers of the athletes left on the basketball, including the velocity and direction. Fig. 2 uses $X^{\prime}, Y$ and $\mathrm{Z}$ as $3 \mathrm{D}$ coordinates to describe the motion model of the basketball. The $\mathrm{X}^{\prime}$ axis represents the horizontal direction from hand to the basketball rack, and the $\mathrm{Y}$ axis represents the moving height of the basketball. It can be assumed that the deflection-angle of the $X^{\prime}$ axis and the direction of the actual shooting in $X^{\prime} Z$ plane is a. Assuming the $\mathrm{Xk}$ axis is the projection of the basketball's flying parabolic path in the $\mathrm{X}^{\prime} \mathrm{Z}$ plane, the following is analysis of $\mathrm{XkY}$, which is as shown in Figure 1:

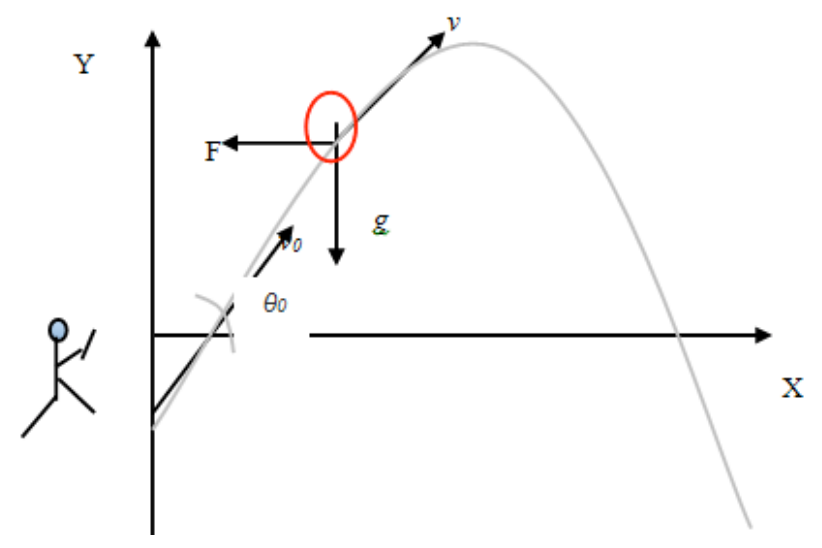

Figure 1. Parabolic Motion

Virtual 3D shooting teaching regards the shooting as an independent and complete system, using the information feedback function of the system to correct errors in each exercise in a timely fashion, which can make the action more and more reasonable. The system can evaluate from the initial disordered state to the ordered state to reach a new equilibrium state, namely the action can form a dynamic stereotype. In the evolution process, the exercisers (students) are always controlling the technique shooting action, so the shooting action can be completed according to the set procedures.

Through this kind of teaching method, it can help students understand shooting techniques from the rational level and then use the rational understanding to guide 
practical exercises. The perceptual understanding from the practical experience can inspect the former rational understanding in turn to obtain the rational knowledge of a standing shot, using the acquired rational standing shot knowledge to guide the practical standing shot technology. In the conventional teaching of single-handed basketshooting, students began learning by imitation. The wrong action also occurred from imitation, but the students did not know where they were wrong. They also did not know how to correct the errors. Correcting only through feedback from the teachers is often not timely and is low in efficiency, which creates the waste phenomenon of recorrecting wrong actions after a correction. Therefore, after the experiment, through the retesting of singlehanded basket shooting of the two classes of students, it captured the hit rate and the technique assessment data to compare the results of the analysis.

\section{THE EXPERIMENTAL RESULTS}

\section{A. Result Analysis of Standing Shot Technique Evaluation and Hit Rate of the Two Classes before the Experiment}

During the process of implementing 3D directional shooting teaching methods and through testing of the students' hit rates of shooting and the scores of the shooting technique before the experiment, we could understand the basic situation of the students' technique level before the experiment. In the shooting teaching process of the experimental class, the objective of each student's shooting technique learning was set with a specific plan according to their situations. The total objective was to master the standing shot technique and use it more skillfully. During the experimental period of each basketball lesson, all students were set with specific shooting skills as the learning objectives, such as completing the shooting exercise, the exercise intensity and the index of the hit rate, etc.

\section{B. Result Analysis of the Hit Rate of Standing Shot of the Two Classes before the Experiment}

Before the experiment, both classes were tested for the hit rate of the standing shot, respectively, by using singlehanded basket shooting at the free throw line position. Each threw the basketball 10 times, remembering the number of baskets; the results are shown in Figure 2.

Fig. 2 shows the distribution and trend of the two polygonal lines, which basically reflect the hit rate of the standing shot of the two classes before the experiment as well as the situation of grasping basketball sports and shooting skills. From the analysis shown in Table I, the hit rate of the standing shot of the two classes before the experiment had no significant differences; the two classes were at the same level. Before the experiment, the hit rate of the standing shot of the control class was $30 \%$, while the experimental class was $28 \%$, respectively, which reflects that the two classes of students had not mastered the standing shot skills and their level of basketball was not very high.

TABLE I.

THE COMPARISON OF THE Hit RATE OF STANDING SHOt OF THE TwO CLASSES BEFORE THE EXPERIMENT $(\mathrm{N}=40)$

\begin{tabular}{|l|c|c|c|c|}
\hline & X & S & T & P \\
\hline The controlled class & 3.1 & 1.3 & 0.415 & $>0.05$ \\
\hline The experimental class & 2.7 & 0.8 & & \\
\hline
\end{tabular}

\section{CONCLUSION}

This thesis proposed a model of parabolic motion focused on basketball, realizing the animation of a basketball after being thrown in the air. In future work, teachers should combine the model of parabolic motion and the algorithm of elastic deformation to simulate possible real shooting situations during the process of shooting, thereby realizing a real simulation of basketball.

\section{REFERENCES}

[1] Hui-gang Wang. The principle and application of computer simulation. National Defense University of Science And Technology Press, 2004.

[2] Yanguo Dong. Computer simulation technology applied in the field of sports teaching. Sports Research.68-70.2008(5).

[3] Xiao-dong Luo. Virtual reality technology and its application in teaching. Education Technology.53-54.2003 (8).

[4] Yong-gang Li.3D face modeling method based on plane surface. Computer Engineering and Design, 157-159.2009(5).

[5] Zhan-yi Hu. Camera calibration method based on active vision. Journal of computers.149-156.2002 (11).

[6] Jian Chen. Motion capturing technology and its application in sports technique diagnosis. Journal of Shanghai Sports Institute.46-49.2006 (4).

\section{AUTHORS}

CHEN Gong is studying for a doctorate degree at $\mathrm{Wu}-$ han University of Technology. He is working at Guangxi Economic \& Trade Polytechnic, Guangxi, CO 530021, China.

CHEN Ning is with Wuhan Textile University, Wuhan, CO430000, China

Submitted 17 September 2015. Published as resubmitted by the authors 23 January 2016.

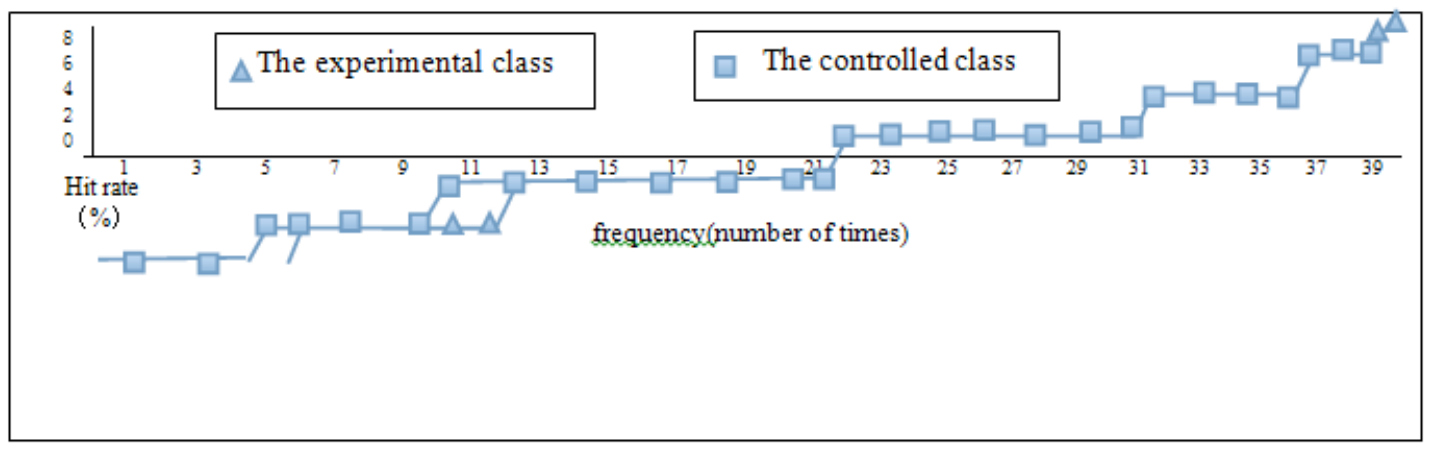

Figure 2. The Situation of the Hit Rate of Standing Shot of the Two Classes before the Experiment 